Dermatology 2012;225:127-130

DOI: $\underline{10.1159 / 000342181}$

\section{Squamomelanocytic Tumor of the Nail Unit Metastasizing to a Sentinel Lymph Node: A Dermoscopic and Histologic Investigation}

Holger A. Haenssle, Timo Buhl, Ricarda Holzkamp,

Department of Dermatology, Venereology and Allergology, Georg August University Göttingen, Göttingen, Germany

\section{Key Words}

Squamomelanocytic tumor $\cdot$ Nail unit $\cdot$ Collision tumor • Melanoma $\cdot$ Squamous cell carcinoma $\cdot$ Dermoscopy

\section{Introduction}

Squamomelanocytic tumor (SMT) is a true malignant cutaneous proliferation composed of closely intermingled cells of squamous cell carcinoma (SCC) and melanoma. Since the publication of a first case series in 1999 [1] only 9 more cases have been reported in the world literature [2-9]. The large majority of earlier cases (11 out of 12) involved chronically sun-exposed skin of the face and scalp area.

A lack of consensus about the proper terminology has led to an interchangeable usage of different expressions. In a more precise interpretation SMT has to be differentiated from a 'collision' of a well-demarcated SCC and melanoma, where the two cell populations are not truly intimately mixed. In contrast to SMT, a biphenotypic malignant proliferation [10] is characterized by tumor cells that arise from a common precursor and stain for markers of both lineages in the same tumor cells (e.g. S-100 and keratin). Moreover, SMT should not be confused with a reactive colonization of only one malignant proliferation with another cell type, e.g. pseudocarcinomatous hyperplasia in melanoma [11] or colonization of SCC with cytologically unremarkable melanocytes [12]. Noteworthy, a case with two concurrent neoplasms involving different nails of the same hand has been reported [13].

We report a first SMT case involving the nail unit of the same finger, which metastasized to the sentinel lymph node. Clinical and dermoscopic aspects, as well as results of an in-depth histopathologic investigation, are presented.

\section{Case Report}

A 75-year-old man presented with an asymptomatic dystrophy of his right thumbnail. He had first noticed changes in nail morphology 2 years earlier and was repeatedly treated for subungual viral warts. There was no family or personal history of melanoma or nonmelanoma skin cancer. The clinical examination (fig. 1a) revealed a complete dystrophy of the nail plate with a multicolored hyperkeratosis of the nail bed (white-yellow, pink, darkgray). The periungual skin showed a faint gray-purple discoloration and areas of prominent brown pigmentation. Hyperkeratotic cysts were macroscopically visible.

Dermoscopy of the periungual skin identified areas with irregular diffuse gray-brown pigmentation (fig. $1 \mathrm{~b}$, box 1) which has been described as a key criterion for acrolentiginous melanoma [14]. White-yellow keratin cysts that are usually found in
Fig. 1. Clinical and dermoscopic presentation of an SMT of the nail unit. a Complete dystrophy of the nail plate with a multicolored hyperkeratotic tumor (white-yellow, pink, dark-gray). Boxes 1-3 indicate areas of dermoscopic images, which are depicted in $\mathbf{b}$. b Box 1 shows a dermoscopic view with diffuse gray-brown pigmentation and keratin cysts, box 2 depicts multiple brown dots, box 3 shows a melanocytic fibrillar pattern combined with dotted/ short linear vessels.

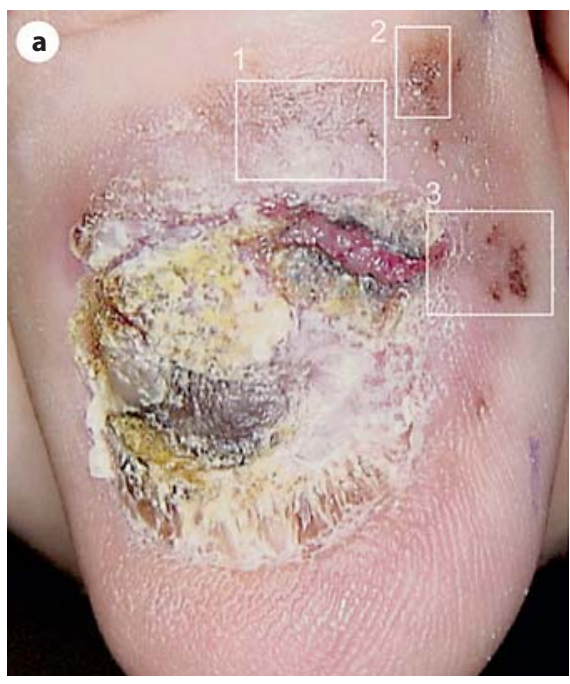

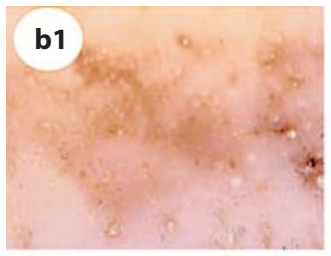

b2

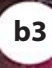

b3

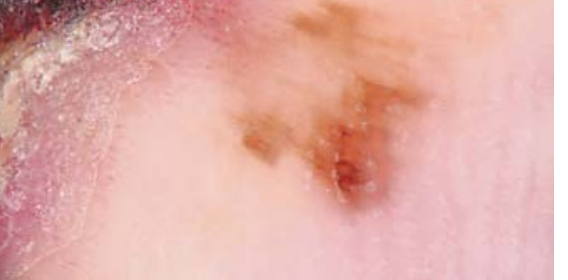

\section{KARGER}

(c) 2012 S. Karger AG, Basel

Fax +41613061234

E-Mail karger@karger.ch

www.karger.com
Accessible online at:

www.karger.com/drm
PD Dr. H.A. Haenssle

Department of Dermatology, Venereology and Allergology

Georg August University Göttingen, Robert-Koch-Strasse 40

DE-37075 Göttingen (Germany)

E-Mail h.haenssle@med.uni-goettingen.de 
Table 1. Published cases of SMTs since the first definition of this entity in 1999

\begin{tabular}{|c|c|c|c|c|c|c|}
\hline 1 & $70 / \mathrm{M}$ & medial canthus & 2.7 & - & 1 year & Pool et al. [1], 1999 \\
\hline 2 & $50 / \mathrm{M}$ & eyebrow & 2 & - & 2 years & Pool et al. [1], 1999 \\
\hline 3 & $44 / \mathrm{F}$ & forehead & 1.9 & - & 9 years & Pool et al. [1], 1999 \\
\hline 4 & $47 / \mathrm{M}$ & nose & 1.0 & - & 1 year & Pool et al. [1], 1999 \\
\hline 5 & $61 / \mathrm{M}$ & preauricular & - & - & 4 years & Dorić et al. [3], 2008 \\
\hline 8 & $68 / \mathrm{M}$ & temple & - & - & - & Leonard et al. [5], 2009 \\
\hline 9 & $62 / \mathrm{M}$ & ear & 2.1 & negative & 9 months & Pouryazdanparast et al. [7], 2009 \\
\hline 10 & $82 / \mathrm{F}$ & nose & 0.75 & - & - & Miteva et al. [6], 2009 \\
\hline 11 & $32 / \mathrm{F}$ & arm & 4.3 & micrometastasis & 10 months & Amerio et al. [2], 2011 \\
\hline 12 & $80 / \mathrm{M}$ & temple & 2.04 & - & 12 months & Scruggs et al. [9], 2011 \\
\hline 13 & $65 / F$ & nose & 1.7 & negative & 12 months & Scruggs et al. [9], 2011 \\
\hline
\end{tabular}

Breslow $=$ Breslow tumor thickness of melanoma portion; $F=$ female; $M=$ male; $F U=$ follow-up.

keratinizing tumors like SCCs or seborrheic keratoses were visible along the proximal eponychium (fig. 1b, box 1). Other dermoscopic criteria clearly associated with melanocytic lesions were also present and included multiple brown dots (fig. 1b, box 2) and a melanocytic fibrillar pattern combined with dotted/short linear vessels (fig. 1 b, box 3 ).

Histology of a punch biopsy from the fibrillar pattern area revealed melanoma in situ. Therefore, the nail apparatus was removed en bloc with wide margins. As demonstrated in longitudinal sections (fig. $2 \mathrm{a}$ ), the tumor extended from the posterior nail fold (fig. 2b) to the epithelium of the nail plate (fig. 2d). A central large irregular bulbous protrusion was confined to the nail matrix (fig. 2c). High-power field examination revealed two distinct and closely intermingled cell populations. Anastomosing epithelial cords and strands with formation of squamous pearls were admixed with atypical epithelioid melanocytic cells grouped in small nests (fig. 2e). The synopsis of all of these findings gave rise to the diagnosis of SMT, i.e. an ulcerated acrolentiginous melanoma (Breslow thickness $4.2 \mathrm{~mm}$ ) intimately admixed with SCC cells.

This diagnosis was confirmed by immunohistochemical analyses (fig $2 \mathrm{f}-\mathrm{h}$ ). Melanoma cells stained positively for markers S-100, Melan-A and HMB-45. A nuclear pattern of reactivity was found for microphthalmia-associated transcription factor. Melanoma cells showed high mitotic activity $\left(>4\right.$ mitoses $\left./ \mathrm{mm}^{2}\right)$ and were found in intimate admixture with squamoid cells uniformly expressing keratin, as revealed by labeling with the pancytokeratin antibody MNF116 (detection of keratins 5, 6, 8, 17 and 19).

Staging procedures including CT scan and ultrasound examination showed no evidence of dissemination. An axillary sentinel lymph node biopsy revealed one subcapsular melanoma micrometastasis $(<0.1 \mathrm{~mm})$ in one of two sentinel nodes. Given that patients with small $(<0.1 \mathrm{~mm})$ sentinel node micrometastases have an excellent long-term prognosis (estimated 5-year overall survival $=91 \%$ ) [15], no complete lymph node dissection was performed after informed consent of the patient. During 24 months of follow-up according to current melanoma guidelines, no evidence of recurrence or metastases was found.

\section{Discussion}

We present clinical, dermoscopic and histologic features of an SMT, a rare tumor with closely intermingled cells of invasive melanoma and SCC. To our knowledge, among the 13 cases in the world literature [1-9], this is the first reported SMT involving the nail unit (table 1).

The clinical and dermoscopic examination revealed features of advanced acrolentiginous melanoma (destruction of the nail unit, multiple colors, atypical vascular pattern, melanoma cells invading the adjacent skin in a fibrillar pattern) as well as subungual SCC (pronounced hyperkeratosis of nail bed, keratin cysts in the periungual skin). The dermoscopic features gave rise to the suspicion for a tumor combined of a melanoma and an SCC. However, a final diagnosis in melanocytic lesions suspicious for melanoma should be made by dermatopathology that still represents the diagnostic 'gold standard' [16].

Histologically, the tumor showed a true malignant proliferation of melanoma cells closely intermingled with SCC cells. The distinct immunohistochemical profile, with none of the tumor cells displaying overlapping markers of both lineages, supported this interpretation and ruled out a true biphenotypic malignant population from a common precursor cell [10]. Given that the two types of tumor cells were intimately admixed, a collision of two distinct tumors or formation of a metastasis of one tumor into the other appeared highly unlikely. In contrast to a simple colonization of SCCs with melanocytes [12], which are cytologically unremarkable and show small nuclei, melanocytes in our case revealed the typical architecture of an aggressive neoplastic proliferation with a malignant, epithelioid cytomorphology and a high mitotic activity. On the other hand, identification of atypical 

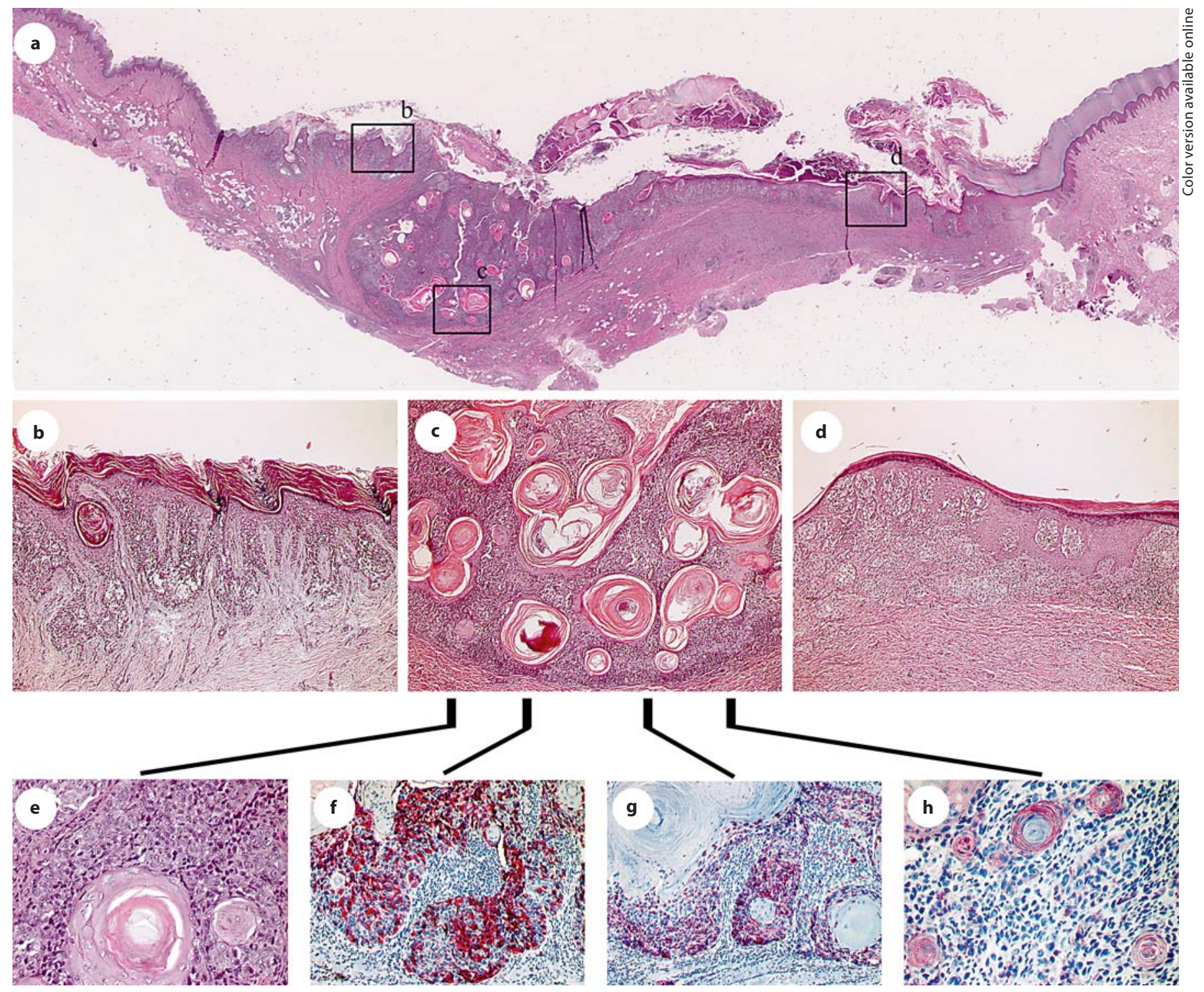

Fig. 2. Histopathology and immunohistochemistry of SMT. a Longitudinal section through the completely removed nail unit. b Melanoma in situ of the posterior nail fold. HE. $\times 40$. c Irregular bulbous projection of the collision tumor confined to the nail matrix containing neoplastic epithelial cords with prominent squamous pearls, admixed with grouped atypical melanocytes. HE. $\times 40$. $\mathbf{d}$ Invasive malignant melanoma of the nail bed below

the fragmented nail plate. HE. $\times 40$. e Higher magnification detail of melanoma cells admixed with squamous pearls. HE. $\times 100$. f HMB-45 immunoreactive atypical melanocytes scattered throughout the SCC. $\times 100$. g Atypical melanocytes coexpress Melan-A. $\times 100$. h Neoplastic squamous cells expressing cytokeratins. Clone MNF116. $\times 100$.

squamoid cells with mitoses, hyperchromatic atypical nuclei, prominent nucleoli, and pronounced dyskeratosis were consistent with the diagnosis of SCC, ruling out pseudocarcinomatous hyperplasia, which has been described as a reactive process in melanomas [11].

Although metastasis has not been reported in most known cases of SMT, a sentinel node micrometastasis of a lesion with a Breslow thickness of $4.3 \mathrm{~mm}$ was described recently [2], and one

of two sentinel nodes of our patient showed a subcapsular melanoma micrometastasis. Thus, although the excellent long-term prognosis of such small metastases of melanoma did not necessitate further therapeutic action in our case [15], lymph node metastasis of SMT is quite possible. Consequently, in the absence of prospective data with this rare tumor, we propose the adherence to current melanoma guidelines in cases of SMT. 


\section{Acknowledgement}

This work was partially supported by a grant from the Niedersächsische Krebsgesellschaft e.V., Hannover, Germany. H.A.H. was supported by a grant from the German Federal Ministry of Research and Technology (ZIM-KOOP program, grant No. KF2111209FR1). The FotoFinder dermoscope used in this study is a donation of FotoFinder Systems GmbH (Bad Birnbach, Germany) to the University Medical Center of Göttingen.

\section{Disclosure Statement}

The authors have no relevant financial interest in this article.

\section{References}

1 Pool SE, Manieei F, Clark WH Jr, Harrist TJ: Dermal squamo-melanocytic tumor: a unique biphenotypic neoplasm of uncertain biological potential. Hum Pathol 1999;30:525-529.

-2 Amerio P, Tracanna M, Di Rollo D, Magnasco S, Angelucci D, Tulli A, Auriemma M, Carbone A: Metastasizing dermal squamomelanocytic tumour. J Eur Acad Dermatol Venereol 2011;25:489-491.

-3 Dorić M, Radovic S, Kuskunovic S, Hukic A, Babic M, Tomic I, Selak I: Dermal squamomelanocytic tumor: neoplasm of uncertain biological potential. Bosn J Basic Med Sci 2008;8:152-155.

$\checkmark 4$ Falanga V, Chartier M, Butmarc J, Tibbetts L: Collision of desmoplastic-neurotropic melanoma and squamous cell carcinoma on the lip. J Cutan Pathol 2008;35:473-476.

5 Leonard N, Wilson N, Calonje JE: Squamomelanocytic tumor: an unusual and distinctive entity of uncertain biological potential. Am J Dermatopathol 2009;31:495-498.

6 Miteva M, Herschthal D, Ricotti C, Kerl H, Romanelli P: A rare case of a cutaneous squamomelanocytic tumor: revisiting the histogenesis of combined neoplasms. Am J Dermatopathol 2009;31:599-603.

7 Pouryazdanparast P, Yu L, Johnson T, Fullen D: An unusual squamomelanocytic tumor of uncertain biologic behavior: a variant of melanoma? Am J Dermatopathol 2009;31:457-461.
8 Rongioletti F, Baldari M, Carli C, Fiocca R: Squamomelanocytic tumor: a new case of a unique biphenotypic neoplasm of uncertain biological potential. J Cutan Pathol 2009;36:477-481.

-9 Scruggs JM, Rensvold EA, Parekh PK, Butler DF: Cutaneous collision cancers: a report of two squamomelanocytic malignancies and review of the literature. Dermatol Surg 2011;37:1679-1683.

10 Rosen LB, Williams WD, Benson J, Rywlin AM: A malignant neoplasm with features of both squamous cell carcinoma and malignant melanoma. Am J Dermatopathol 1984;6(suppl):213-219.

11 Mott RT, Rosenberg A, Livingston S, Morgan MB: Melanoma associated with pseudoepitheliomatous hyperplasia: a case series and investigation into the role of epidermal growth factor receptor. J Cutan Pathol 2002;29:490-497.

12 Hara K, Kamiya S: Pigmented eccrine porocarcinoma: a mimic of malignant melanoma. Histopathology 1995;27:86-88.

13 Harwood M, Telang GH, Robinson-Bostom L, Jellinek N: Melanoma and squamous cell carcinoma on different nails of the same hand. J Am Acad Dermatol 2008;58:323-326.

14 Saida T, Miyazaki A, Oguchi S, Ishihara Y, Yamazaki Y, Murase S, Yoshikawa S, Tsuchida T, Kawabata Y, Tamaki K: Significance of dermoscopic patterns in detecting malignant melanoma on acral volar skin: results of a multicenter study in Japan. Arch Dermatol 2004;140:12331238.

15 Van Akkooi AC, Nowecki ZI, Voit C, Schafer-Hesterberg G, Michej W, de Wilt JH, Rutkowski P, Verhoef C, Eggermont AM: Sentinel node tumor burden according to the Rotterdam criteria is the most important prognostic factor for survival in melanoma patients: a multicenter study in 388 patients with positive sentinel nodes. Ann Surg 2008;248: 949-955.

16 Braun RP, Gutkowicz-Krusin D, Rabinovitz H, Cognetta A, HofmannWellenhof R, Ahlgrimm-Siess V, Polsky D, Oliviero M, Kolm I, Googe P, King R, Prieto VG, French L, Marghoob A, Mihm M: Agreement of dermatopathologists in the evaluation of clinically difficult melanocytic lesions: how golden is the 'gold standard'? Dermatology 2012;224: $51-58$. 